\title{
Phase closure nulling
}

\section{Application to the spectroscopy of faint companions}

\author{
A. Chelli, G. Duvert, F. Malbet, and P. Kern \\ Laboratoire d'Astrophysique de Grenoble and Mariotti Center, UMR 5571 Université Joseph Fourier, BP 53, \\ 38041 Grenoble Cedex 9, France \\ e-mail: Alain.Chelli@obs.ujf-grenoble.fr
}

Received 25 September 2008 / Accepted 16 December 2008

\section{ABSTRACT}

\begin{abstract}
We provide a complete theory of the phase closure of a binary system in which a small, feeble, and unresolved companion acts as a perturbing parameter on the spatial frequency spectrum of a dominant, bright, resolved source. We demonstrate that the influence of the companion can be measured with precision by measuring the phase closure of the system near the nulls of the primary visibility function. In these regions of phase closure nulling, frequency intervals always exist where the phase closure signature of the companion is larger than any systematic error and can then be measured. We show that this technique allows retrieval of many astrophysically relevant properties of faint and close companions such as flux, position, and in favorable cases, spectrum. We conclude by a rapid study of the potentialities of phase closure nulling observations with current interferometers and explore the requirements for a new type of dedicated instrument.
\end{abstract}

Key words. stars: fundamental parameters - stars: planetary systems - stars: binaries: close - stars: binaries: spectroscopic instrumentation: interferometers - methods: observational

\section{Introduction}

A common feature of the observational problem posed by spectroscopic binaries, evolved stars companions, hot Jupiters, and extrasolar planets is the presence of a point-like source of low flux in the vicinity of a much larger and much brighter star. Indeed, it is the properties of the faint companion that are desired, and are the most difficult to acquire. Directly detecting the flux of an extrasolar planet and establishing its spectrum seems the most demanding task, to be fulfilled only by dedicated spatial missions (DARWIN: Cockell et al. 2008, and TPF-I: Lawson et al. 2008) using interferometry nulling techniques (Bracewell 1978).

Ground-based optical long baseline interferometers also have the capability of detecting extrasolar planets restricted to the case of a giant planet orbiting close to its parent star (Vannier et al. 2006), such as 51 Peg the first exoplanet to be unraveled by radial velocity techniques (Mayor \& Queloz 1995). The principle consists in measuring interferograms in different spectral regions where the ratio between the planet photons and the stellar ones changes because of an emission line or an absorption line in the planet atmosphere. The position of the interferogram measured by the phase is directly related to the position of the photocenter of the star-planet system on the sky. The slight shift in the phase between two spectral regions is in principle measurable for bright extrasolar planets such as hot Jupiters.

Even when using color-differential techniques the phase measurement is plagued by the variation of the refractive index in air. This is why efforts are currently concentrated on using the phase closure, which corresponds to the sum of the phases measured in three connected baselines (see Sect. 2.4 for details). This technique allows the observer to remove any atmospheric perturbation or instrumental-based errors localized in each arm of the interferometer. This detection has not been performed yet because of instrumental biases and the need to decrease the photon noise even with large telescopes.

An important property of stars resolved by long baseline interferometry is that the coherence of the light decreases with increasing spatial frequencies down to zero before increasing again following the well known behavior of the Bessel functions. Michelson \& Pease (1921) used this property to measure the diameter of Betelgeuse for the first time. Indeed, the visibility of a photosphere of uniform brightness distribution over a circular disk is canceled out at the spatial frequency $0.61 / R_{\star}$ where $R_{\star}$ is the stellar angular radius. Another property is that the phase of the visibility jumps by 180 degrees at each crossing of the Bessel function's zeros (hereafter called visibility nulls or simply nulls). At each null, the coherent flux of the star is canceled out, making it easier to detect a change in the phase jump due to a faint close companion.

In this paper we demonstrate that, in precisely the restricted case of the lobe crossing, the influence of even a very faint companion can be measured with precision, and its spectrum can be obtained by measuring the phase closure of the system near the nulls of the primary visibility function. In these regions where the phase closure nulling of the primary is effective, the phase closure signature of the secondary stands out to a point where its basic properties (flux, position, spectrum, etc.) can be measured. In a companion paper (Duvert et al. 2009), this technique was used to detect the companion of the single-lined spectroscopic binary $\sigma$ Puppis, at a few stellar radii distance.

\section{Description of the problem}

\subsection{Visibility of a stellar photosphere of uniform brightness}

When observing a star with a multi-aperture interferometer, it is well known that one detects the amplitude of the visibility 

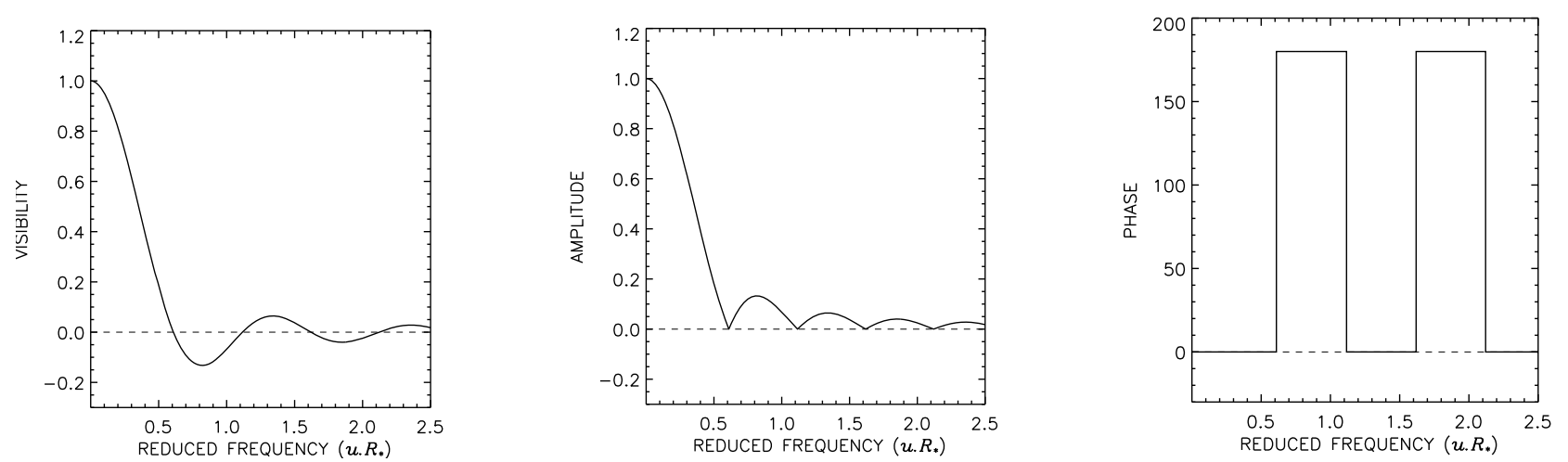

Fig. 1. Visibility for a star represented well by a uniform brightness distribution over a disk of angular radius $R_{\star}$ (left panel), as a function of the reduced frequency (defined as the frequency times the stellar radius). The visibility is often presented in the form of amplitude (center panel) and phase (right panel).

function (or the amplitude squared) and that this amplitude decreases with increasing spatial frequency $u=B / \lambda$, where $B$ is the baseline and $\lambda$ the wavelength. Thanks to the Zernike-Van Cittert theorem, it can be shown that this visibility function coincides with the Fourier transform of the spatial energy distribution of the star; hence, for a star whose energy distribution is well-represented by a uniform disk, the visibility function is proportional to a Bessel function of the first order

$V_{\star}(u)=2 \frac{J_{1}\left(2 \pi u R_{\star}\right)}{2 \pi u R_{\star}}$.

The visibility function is a complex number that, in this special case of a simple centro-symmetric brightness distribution, turns out to be real (i.e. the imaginary part is zero). It is often displayed by its amplitude and its phase (see Fig. 1).

The visibility becomes negative when the baseline is longer than $0.61 \lambda / R_{\star}$, corresponding to the first null, so the phase suddenly changes from zero to 180 degrees (see right panel of Fig. 1). It becomes positive again when crossing the second null, and so on.

\subsection{Visibility of a resolved star with a faint companion}

When an object is composed of two components, the object visibility function is the sum of the visibility function of the two components weighted by the fraction of flux of each component and a phase modulated by the apparent angular separation on the sky. If one adds a faint companion to the star of the previous section with a flux ratio $r$ and separated from the star by $s$ (assuming for sake of simplicity that the system orientation is parallel to the frequency axis), then the complex visibility of the system is

$\hat{\imath}(u)=\frac{V_{\star}(u)+r \mathrm{e}^{\mathrm{i} 2 \pi u s}}{1+r}$.

If the flux ratio $r$ is small, the amplitude of the visibility function will be very slightly modified by the presence of the faint companion. The stronger effect occurs around visibility nulls of the primary, where the system visibility is no longer zero. At these locations, the visibility perturbation is equal to the flux ratio. This effect still remains weak and is beyond the performances of current interferometers as soon as the flux ratio is less than about $1 \%$. However, besides the fact the companion is not detectable, studies around visibility nulls keep their interest for precise diameter and limb darkening measurements (Le Bouquin et al. 2008).

\subsection{Phase changed by the faint companion}

More interesting is the phase of the object visibility. By definition, the tangent of the phase of a complex number is equal to the ratio of its imaginary part by its real part. Therefore we have

$$
\tan \phi(u)=\frac{r \sin (2 \pi u s)}{V_{\star}(u)+r \cos (2 \pi u s)} .
$$

For small flux ratios and except for around nulls, the phase of the object is very close to 0 or 180 degrees, and may be approximated by

$\phi(u) \approx \frac{r \sin (2 \pi u s)}{V_{\star}(u)}+n \pi$

with $n=0$ if $V_{\star}(u)>0$, and $n=1$ if $V_{\star}(u)<0$. For separations $s / R_{\star}$ much greater than 1 , it is quasi periodic, with a pseudo period $s^{-1}$.

The phase is the result of two contributions: from the star $V_{\star}(u)$ and from the companion $r \mathrm{e}^{\mathrm{i} 2 \pi u s}$. The contribution of the star decreases as the frequency increases and is even canceled at the nulls, while that of the companion keeps constant as long as it is not resolved. Most of the time, the companion produces a phase signature in the range $\pm r / V_{\star}(u)$. But more important, around visibility nulls, in the frequency ranges for which $\left|V_{\star}(u)\right| \leq r$, this phase signature becomes significant, with an exact value of $2 \pi u_{0} s$ at the frequencies $u_{0}$ of the nulls $\left(\approx \pi s / R_{\star}\right.$ for the first null), much greater than 180 degrees. It follows that, as opposed to the visibility amplitude, even for small flux ratios, frequency intervals always exist around nulls, where the phase signature of the companion is larger than any systematic error and can then be measured.

\subsection{Phase closure of a resolved star with a faint companion}

An interferometer formed by 3 telescopes transmits 3 frequency systems, $u_{12}, u_{23}$, and $u_{13}=u_{12}+u_{23}$, associated with the 3 baselines, each characterized by a phase and a visibility. It provides one phase closure defined as the phase of the bispectrum $\hat{I}\left(u_{12}, u_{23}\right)$, with

$\hat{I}\left(u_{12}, u_{23}\right)=\left\langle\hat{1}\left(u_{12}\right) \hat{1}\left(u_{23}\right) \hat{1}^{*}\left(u_{13}\right)\right\rangle$

where * denotes the complex conjugate, and \langle\rangle represents an ensemble average. The phase closure has two important properties: it is insensitive to atmospheric phase fluctuations, and for a 


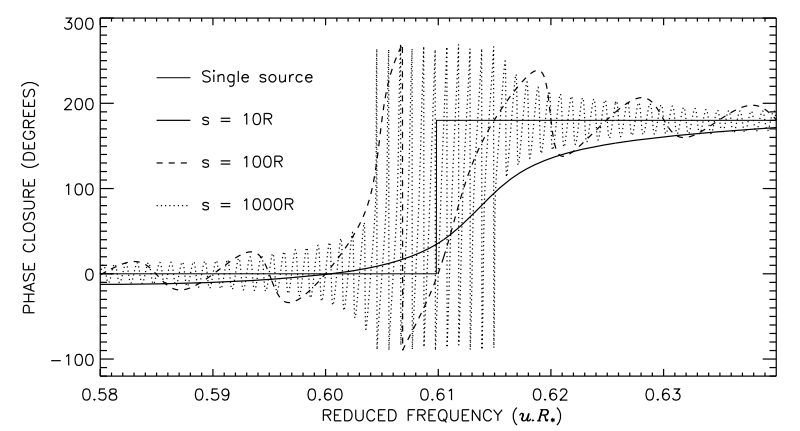

Fig. 2. Phase closure modulo $2 \pi$ of a double system, formed by an extended uniform disk and a point source, as a function of the maximum reduced frequency. The spectral resolution is $\mathcal{R}=1500$. The 3 frequencies $\left\{u_{23}, u_{12}, u_{13}\right\}$ (see Eq. (5)) have been chosen in the ratio 1, 2, 3, with the maximum frequency around the first zero of visibility of the primary star. The phase closure is displayed for 3 separations $\left(10 R_{\star}\right.$, $100 R_{\star}$, and $1000 R_{\star}$ ) and a flux ratio of 0.01 . The signature of the secondary source is dominant in the region around the minimum visibility of the primary (near reduced frequency 0.61 ). The thin line with a 180 degree shift at 0.61 corresponds to the phase closure of the primary alone.

point source, it is equal to zero. Then, for an extended object, it is given by

$\phi_{\mathrm{c}}=\phi_{\mathrm{o}}\left(u_{12}\right)+\phi_{\mathrm{o}}\left(u_{23}\right)-\phi_{\mathrm{o}}\left(u_{13}\right)$

where $\phi_{\mathrm{o}}$ is the phase of the object spatial Fourier transform.

If the telescopes are aligned with baselines parallel to the direction of the double system, the phase closure may be written

$$
\begin{aligned}
& \phi_{\mathrm{c}} \approx \frac{r \sin \left(2 \pi u_{12} s\right)}{V_{\star}\left(u_{12}\right)}+\frac{r \sin \left(2 \pi u_{23} s\right)}{V_{\star}\left(u_{23}\right)}-\frac{r \sin \left(2 \pi u_{13} s\right)}{V_{\star}\left(u_{13}\right)} \\
&+\left(n_{12}+n_{23}-n_{13}\right) \pi .
\end{aligned}
$$

Figure 2 shows the expected phase closure, around the first visibility null, from a double system with a flux ratio of $1 \%$ and various separations. The system is observed with a spectral resolution of 1500, like the one available with the AMBER instrument at the VLTI (Petrov et al. 2007). Note the importance of the phase closure signature from the companion.

\section{Phase closure nulling}

In the case of a system formed by an extended uniform disk and a point source, the useful phase closure signal is the departure from 0 or 180 degrees. We have then clearly interest in selecting the working frequency interval at the highest frequencies, around visibility nulls at $u=u_{0}$, where the phase closure signature of the companion is at its maximum. In these regions, there is always (as the stellar visibility crosses the zero) a frequency interval within which the useful signal is stronger than any systematic error, hence can be measured.

Figure 3 shows the useful phase closure signal for various flux ratios and distances of 10,100, and 1000 stellar radii. The horizontal lines correspond to a given systematic error of 0.1 degree. The frequency interval within which the useful signal is stronger than this error diminishes rapidly with the flux ratio, but is always present.

We propose using the regions around minima of visibility, where the phase closure nulling of the primary is effective, and the useful signal is over the limit $\sigma_{\text {sys }}$ imposed by systematic errors, to detect and to characterize faint companions. By doing

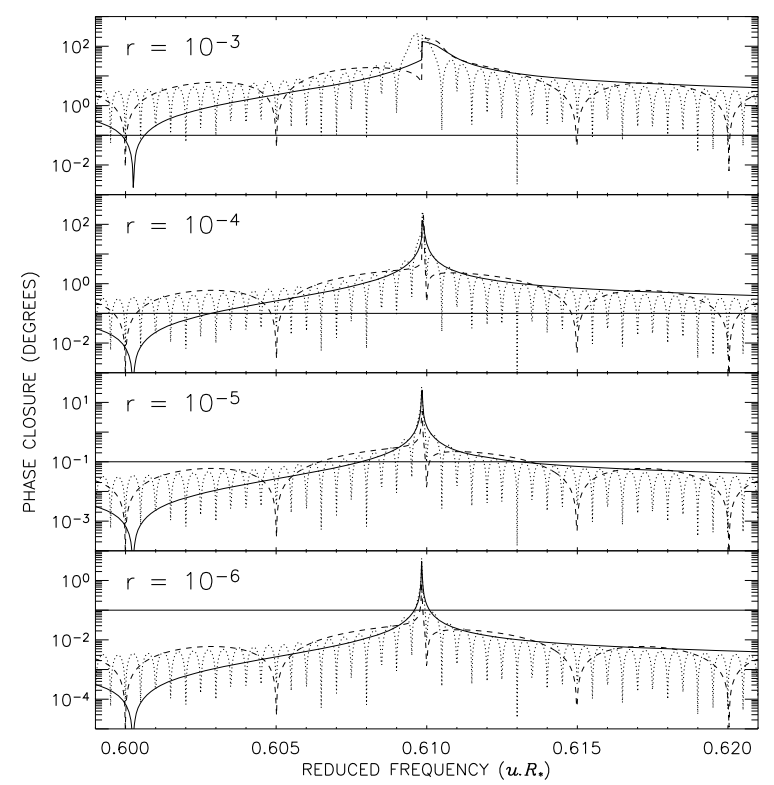

Fig. 3. Absolute value of the useful phase closure signal of a double system formed by a uniform disk and a point source as a function of the highest frequency located around the first visibility null of the primary. The spectral resolution is $\mathcal{R}=1500$. The phase closure of the uniform disk has been subtracted for clarity. From top to bottom, the flux ratio varies from $10^{-3}$ to $10^{-6}$, and 3 separations are considered: $10 R_{\star}$ (full lines), $100 R_{\star}$ (dashed lines), $1000 R_{\star}$ (dotted lines). The horizontal lines are set to a given systematic error of 0.1 degree, showing the regions where the phase closure signature of the secondary becomes measurable.

so, we do not cancel the stellar flux as in classical nulling experiments (Bracewell 1978), but we only cancel the stellar coherent flux. The main limiting noise will then be the photon noise from the central star, as shown in Sect. 3.2.

\subsection{Spectroscopy of close companions}

The spectroscopy of close companions may be extracted from spectrally dispersed phase closure measurements obtained with an $N_{\text {tel }}$ telescopes interferometer $\left(N_{\text {tel }} \geq 3\right)$. The baselines should be such that the longest one corresponds to a null of the primary star in order to maximize the useful signal. As an example, an interferometer with 3 telescopes and frequencies $\left\{u_{23}, u_{12}, u_{13}\right\}$ in the ratio $1: 2: 3$, allowing bootstrapping, would be a very efficient system. The data consists of spectrally dispersed phase closure measurements performed as a function of the spatial frequency.

The double system is characterized by 4 parameters: the stellar radius $R_{\star}$, the flux ratio $r$, the separation $s$, and the position angle. The 4 parameters may be extracted from a modeling of the phase closure variations as a function of both the spatial frequency and the wavelength. The spectrum of the companion is then obtained by multiplying the flux ratio by the spectrum of the primary.

\subsection{Error analysis}

We consider for simplicity an interferometer formed by $N_{\text {tel }}$ aligned telescopes in a non-redundant configuration. We also assume that the direction of observation varies little during the experiment so that the double system is characterized by only 3 parameters: the stellar angular radius $R_{\star}$, the flux ratio $r$, and the projected separation $s$ along the direction of observation. 
Spectrally dispersed phase closure measurements are performed as a function of the spatial frequency and then fitted, with a 3-parameter model.

The limiting noises in optical interferometry from the visible to the near infrared are the atmospheric noise, the photon noise from the source, and the detector readout noise. General expressions of the errors on the parameters of the double system are derived in Appendix A. In the photon noise regime, the errors reach their minimum values and then stay constant as soon as the working frequency domains cover at least one period $s^{-1}$. For separations greater than a few stellar radii and working frequency intervals over $s^{-1}$, the error on the flux ratio and that on the separation, with a 3-telescope interferometer, are given by

$\sigma(r) \approx \frac{3}{\sqrt{K}}$

$\sigma\left(s / R_{\star}\right) \approx \frac{1}{r \sqrt{K}}\left(\frac{0.61}{\bar{u}_{\max } R_{\star}}\right)$

where $K$ is the total number of photoevents detected during the whole observation and $\bar{u}_{\max }$ the average frequency of the longest baseline.

These formulae have been checked numerically through a rigorous error analysis. They are valid everywhere, even around the nulls. Besides the error on the separation being inversely proportional to the maximum frequency, the errors are independent of the working frequency domains. This clearly reinforces the usefulness of observing around visibility nulls, where the useful phase closure signal is dominant. The error on the flux ratio only depends on the total number of collected photoevents, while that on the distance is also inversely proportional to the flux ratio. In the photon noise regime, both errors are independent of the spectral resolution $\mathcal{R}$, as long as the spectral averaging is performed over less than half a period $1 / 2 s$, that is, when $\bar{u}_{\max } \mathcal{R}^{-1}<0.5 s^{-1}$. This condition provides a maximum recoverable distance imposed by the spectral resolution of

$\frac{s_{\max }}{R_{\star}} \approx \mathcal{R} \times\left(\frac{0.61}{\bar{u}_{\max } R_{\star}}\right)$.

The error on the flux of the companion is

$\sigma(r K) \approx 3 \sqrt{K}$.

The direct photometric detection of the companion would provide an error of $\sqrt{r K}$. In terms of performances, the direct detection is $3 / \sqrt{r}$ better than detection from phase closure. However, this is the price to pay because, to our knowledge, there is no other direct method than the one we propose, which is capable of detecting stellar companions within an Airy disk and at a distance from the hosting star as small as a few stellar radii. Once the conditions above are fulfilled, measuring the flux ratio for each spectral element at resolution $\mathcal{R}$ is an effective way to measure the companion spectrum.

\subsection{Performances}

The mean error on the flux ratio in the $H$ band at $1.65 \mu \mathrm{m}$ from a 3-telescope interferometer and an integration time of $3 \mathrm{~h}$, obtained by averaging all the spectral channels over an optical bandwidth of $0.3 \mu \mathrm{m}$, is plotted in Fig. 4a. The upper curves correspond to 3 telescopes of $2 \mathrm{~m}, 1 \%$ transmission, and a Strehl ratio of 0.5 . It roughly represents the present state of the AMBER instrument on the VLTI with Auxiliary telescopes. The middle

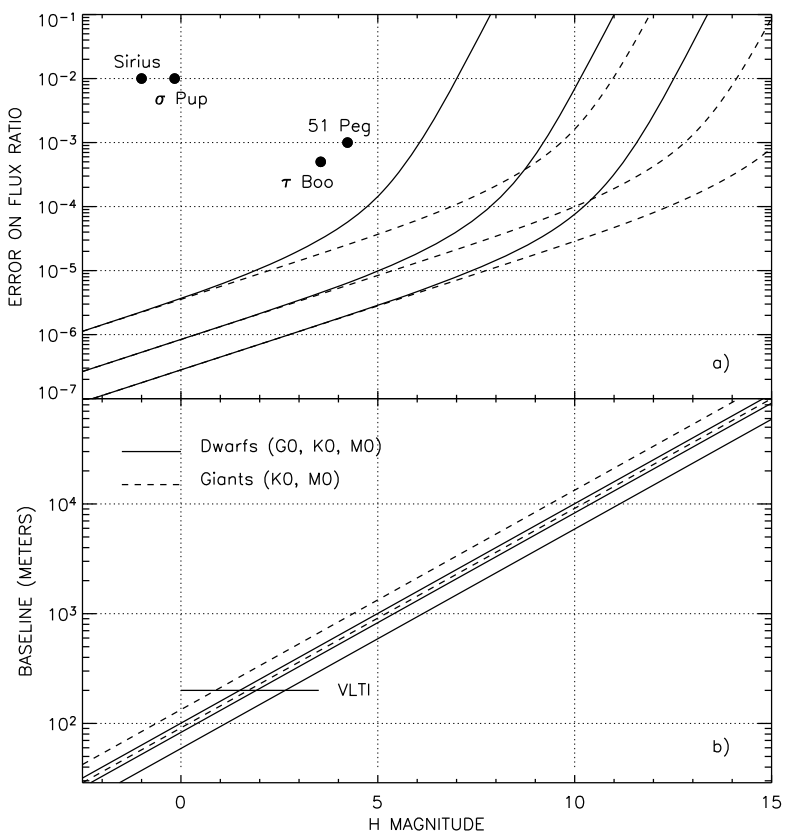

Fig. 4. a) (Top): error on the flux ratio of a double system from the closure phase information of a 3-telescope interferometer. The signal is made by 900 phase closure regularly sampled within the working frequency intervals, and spectrally dispersed along 270 spectral channels, with a spectral resolution of 1500 . The experimental parameters are; observing wavelength: $1.65 \mu \mathrm{m}$; optical bandwidth: $0.3 \mu \mathrm{m}$; total integration time: $3 \mathrm{~h}$; integration time per frame: $0.2 \mathrm{~s}$; detector readout noise: $10 \mathrm{e}^{-} ; 32$ pixels per frame. The calculations took the photon noise and the detector readout noise into account. Three cases were considered: 3 telescopes with diameter $D=2 \mathrm{~m}$, a Strehl ratio $S=0.5$, and $1 \%$ total transmission (upper curve, this case corresponds roughly to the present state of the AMBER experiment on the VLTI), $D=2 \mathrm{~m}$, $S=0.9$ and $10 \%$ total transmission (middle curve), $D=8 \mathrm{~m}, S=0.5$ and $10 \%$ transmission (lower curve). The broken lines correspond to an integration time per frame of $12 \mathrm{~s}$. Represented are: Sirius with a possible M dwarf companion (Benest \& Duvent 1995), the giant K5 star $\sigma$ Puppis with its G2 dwarf companion (Duvert et al. 2009), and two stars with known planetary companions. b) (Bottom): baseline needed to resolve the primary star in the $H$ band at $1.65 \mu \mathrm{m}$, namely to reach the first visibility null, for late dwarfs and giants. The horizontal line at $200 \mathrm{~m}$ corresponds to the maximum baseline of the VLTI.

and lower curves correspond to telescopes of $2 \mathrm{~m}$ and $8 \mathrm{~m}$, a transmission of $10 \%$, and Strehl ratios of 0.9 and 0.5 , respectively (see the caption of Fig. 4 for details).

If we assume a signal-to-noise ratio of 10 on the flux ratio for a positive detection, then the AMBER instrument with Auxiliary telescopes would allow detection of companions $10^{4}$ fainter around second-magnitude stars and $10^{3}$ fainter around fivemagnitude stars. Spectroscopy would be possible from flux ratios of $10^{-4}$. Optimized interferometers with $10 \%$ transmission (Fig. 4, middle and bottom curves) would allow the detection of companions $10^{4}$ fainter around 5 to 7 mag stars and $10^{3}$ fainter around 8 to 10 mag stars. Spectroscopy would be possible from flux ratios of $10^{-5}$.

Figure $4 \mathrm{~b}$ shows the baseline needed to resolve the primary star, that is, to reach the first visibility null for late dwarfs and giants. The horizontal line at $200 \mathrm{~m}$ corresponds to the maximum baseline of the VLTI, which imposes maximum $H$ magnitudes of about 2.5 and 1.5 for M0 dwarves and giants, respectively. Also the field of view of fiber-linked interferometers is limited to one Airy disk $\lambda / D$, where $D$ is the telescope diameter. Given that the baseline for reaching the first null is $B=0.61 \lambda / R_{\star}$, it gives 


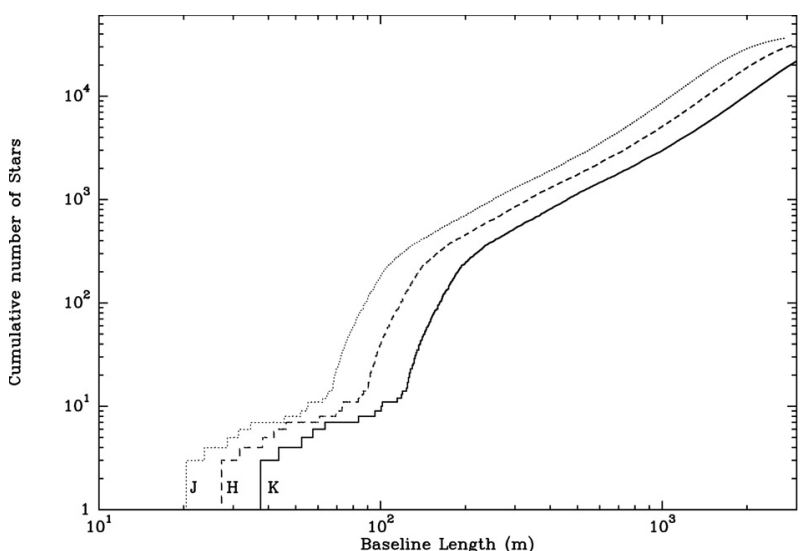

Fig. 5. Lower limit of the number of stars resolved at a given baseline in the $J, H$, and $K$ bands.

a maximum recoverable separation of about $B / D$ stellar radii, which is 100 stellar radii on the VLTI with $2 \mathrm{~m}$ telescopes. A baseline of $1000 \mathrm{~m}$ necessary for resolving a solar type star at a distance of $10 \mathrm{pc}$ would provide a field of 500 stellar radii.

As specified in Sect. 3.2, the minimum error is attained in the photon noise regime when the working frequency intervals cover at least one period $s^{-1}$. This implies, for the first null, a baseline variation $\Delta B$ of

$\Delta B \approx 2 \frac{B}{S / R_{\star}}$.

At kilometric baselines, it represents a variation of $200 \mathrm{~m}$ for a distance of 10 stellar radii. The simplest way to achieve such variations is to use earth rotation with repositionable telescopes located at positions such that the baselines and orientations optimize the observing time around the nulls. However, the double system may also be characterized at the price of a lower signalto-noise ratio, even if the working frequency intervals cover much less than a period. Indeed, the companion of the spectroscopic binary $\sigma$ Puppis has been detected and characterized with only 3 snapshot observations of $5 \mathrm{~min}$ each; before, during and after the null, using the spectral dimension to increase the frequency coverage.

\section{Discussion}

\subsection{Science cases}

We have demonstrated that a phase closure nulling experiment can bring astrophysically significant information on the close environment of resolved stars. Figure 5 shows the lower limit of the number of targets available to phase closure nulling experiments as a function of baseline length. The sample was obtained with the SearchCal utility (Bonneau et al. 2006) and is limited to the stars whose apparent diameter can be reliably estimated from parallax and (spectro)photometric measurements available at the CDS. The minimum number of targets available for current arrays like the VLTI is $\approx 100$ in $K$ and $\approx 500$ in $J$. The technique is not limited to observing unbalanced binary systems but can also address a broader range of topics. These topics may change either by changing the nature of the resolved bright astrophysical target and/or the off-axis accompanying astrophysical signal.

In star and planet formation, astronomers are interested in protoplanetary disks, which in general have the property of being centro-symmetric so that the phase closure signal is zero. Any perturbating signal located off-axis can then be detected by closure phase nulling: inhomogeneities on the disk surface, presence of a forming planetesimal, presence of a collimated jet of outflowing wind partially screened by the disk. It also applies to other science cases holding disk-like cataclysmic variables, active galactic nuclei, proplanetary nebulae, gaseous disk around hot stars. The case of exoplanets is of particular interest, but the various kind of binaries must not be forgotten involving young, main sequence, or evolved stars and even the case of merging binaries.

In a future where interferometry is sensitive to fainter targets, a possible application of this technique is the detection of the gravitational images due to a massive gravitational lens spatially resolved. In the following sections, we restrict ourselves to the companion of evolved and main sequence stars.

\subsubsection{Companions of evolved stars}

Clearly the simplest observational case, already permitted with current interferometers, is the study of close companions to giant stars. The scientific interest is twofold: improving our knowledge of the masses of giant stars and detecting planetary systems around earlier type stars than the ones known to date. It is indeed customary to look for companions of stars of masses $>1.2 R_{\odot}$, not in their main-sequence phase, where radial velocity measurements are rendered difficult by the paucity of spectral lines (furthermore broadened by the rapid stellar rotation), but in their giant, cooler phase. However, the detection of substellar companions around giant stars using radial velocities has always been problematic because the intrinsic pulsation of those stars would mimic the signature of a close substellar companion, and radial profiles can be altered by large, slowly rotating, cool spots on the surface. Only a few detections have been reported to date (Hatzes \& Cochran 1998; Hatzes et al. 2005; Döllinger et al. 2007). The phase closure nulling would provide a completely independent method of characterizing at least the closest companions, and give the orbit needed to measure individual masses.

We demonstrate this very possibility in a companion paper (Duvert et al. 2009), where we apply the phase closure nulling method for the detection of the close companion of the star Sigma Puppis and the characterization of this SB1 binary system.

\subsubsection{Companions of main-sequence stars}

These companions can range from brown dwarves (hereafter BDs) or hot Jupiters (HJs) to earth-like planets. With an expected flux ratio of a few $10^{-4}$ in the near infrared, brown dwarves could be detected with present-day telescope apertures at any distance from the star within the field-of-view permitted $(\approx B / D)$. The experiment could bring two new pieces of information: the mass of the BDs (by solving the orbit) and the NIR spectrum, possibly different in close binaries than the spectra of isolated BDs.

A few HJs, thus very close to their star $\left(<20 R_{\star}\right)$ and with temperatures of $1000-1500 \mathrm{~K}$, will be detectable as well with a flux ratio in the $10^{-4}-10^{-3}$ range in $K$ band and longwards (see e.g. Fortney et al. 2008). A handful of HJs have already been photometrically detected with the IRAC and MIPS instruments of the Spitzer Space Telescope (Charbonneau et al. 2005; Knutson et al. 2008) in transit experiments. Phase closure nulling experiments in this case would permit the NIR spectrum of the object, the orbit and thus the mass to be obtained, without being restricted to transit objects. Phase closure nulling experiments on dwarf stars with HJs would only be possible today 
on a handful of them since the baseline needed to null a Sun equivalent at $10 \mathrm{pc}$ is $\approx 1000 \mathrm{~m}$ at $1.65 \mu \mathrm{m}$. This restriction can be overcome in the near future as fibered interferometers with kilometer baselines are currently underway (Perrin et al. 2006).

For any planetary companions still cooler and smaller than HJs, the flux ratio between the star and the companion rapidly becomes forbidding $\left(>10^{9}\right)$ in the NIR and is manageable only longwards of $10 \mu \mathrm{m}$ (see Fig. 2 in Traub \& Jucks 2002). In the $N$ band, the above-mentioned constraint on the baseline length then becomes very challenging $\left(>10^{4} \mathrm{~m}\right)$, and the signal transmission on such distances would be quite impossible for ground-based interferometers, unless techniques like the parametric conversion to visible or NIR wavelength, such as described in Del Rio et al. (2008), are used before recombination.

\subsection{Towards a dedicated instrument}

We have discussed the main properties of phase closure nulling: around each null of the visibility function of the star, a spatial frequency interval exists where the signal of a companion becomes stronger than any systematic error, hence potentially detectable. The errors on the companion detection, both in flux ratio and in position, depend roughly on the square root of the number of star photons detected, and the imageable region for a fiber-linked interferometer (the distance from the star where the companion's signature can be seen) is $\approx B / D$ stellar radii. The need to be at a star null fixes a minimum baseline length. The need to reach a given sensitivity for the flux ratio implies either a given minimum size for the telescope apertures, thus a maximum imageable area, or/and a given integration time. The spectral resolution $\mathcal{R}$ does not need to be very high, typically $\approx B / D$.

Today interferometers with baselines of a few hundred meters can already detect faint companions to the few closest giant stars using phase closure nulling. It seems possible to go further - and still use ground-based optical arrays - to characterize the faint companions, down to HJ masses, of the nearby mainsequence stars. This can be envisioned with an interferometer, that

1. reaches the first zero of a solar-type star at $10 \mathrm{pc}$ in an infrared band - already kilometric baselines in $H$ band;

2 . has a spectral resolution of $\approx B / D$ to enable detection of companions up to $\approx B / D$ stellar radii. This is 500 for a $1000 \mathrm{~m}$ baseline and $2 \mathrm{~m}$-class telescopes;

3. permits long integration times on the zero crossing thanks to earth rotation and repositionable telescopes;

4. has the highest attainable transmission: since our S/N only depends on the photon $\mathrm{S} / \mathrm{N}$ of the primary, this should be as large as possible. Since we are only interested in phase closure that is a robust measurement insensitive to relay optics wavefront disturbance or atmosphere stability, the attention should go on improving the transmission of the relay optics, not their optical quality. This is best realized with fully integrated optics, fiber linking the foci of the telescopes at the primary focus.

5. uses a few detectors with low noise for the detection, such as a 4-pixel ABCD method. New developments (Rothman et al. 2007) seem to imply that such low-noise IR detectors can be available in a few years from now.

6. takes advantage of the new developments in integrated optics, permitting both beam recombination and spectrography, thus avoiding the losses at all the diopters of bulk optics used for beam transportation and in the spectrograph. Such a concept, derived from on-going developments for visible spectroscopy (Le Coarer et al. 2007) is under investigation (Kern et al. 2009) and can be envision on the sky in the coming few years.

\section{Conclusion}

We have introduced both the concept of phase closure nulling and its application in the detection of faint close companions to resolved stars, accuratly measuring their position and spectra. This concept is the only one to date to permit such direct detection inside an Airy disk. We developed the complete error analysis of the phase closure nulling, and show how this method permits ground-based interferometry to complement and, in favorable cases, compete with the direct detection methods considered for future space-borne experiments. We discussed a few science cases that would benefit from phase closure nulling experiments and sketched the requirements for a new type of interferometer dedicated to such studies.

\section{Appendix A: Error calculation}

\section{A.1. Error on phase closure}

The formal error on the phase closure, in the presence of photon noise and detector readout noise, was computed by Chelli (1989) and Tatulli \& Chelli (2005). Neglecting the coupling between the photon noise and the detector noise and retaining the dominant terms, the variance of the phase closure $\phi_{\mathrm{c}}$ may be approximated by

$$
\begin{aligned}
\sigma^{2}\left(\phi_{\mathrm{c}}\right) \approx & \frac{N_{\mathrm{tel}}^{2}}{2 K} \times\left[\frac{1}{\left|V\left(u_{12}\right)\right|^{2}}+\frac{1}{\left|V\left(u_{23}\right)\right|^{2}}+\frac{1}{\left|V\left(u_{13}\right)\right|^{2}}\right] \\
& +\frac{1}{2}\left(\frac{N_{\mathrm{tel}}^{2} N_{\mathrm{pix}} \sigma_{r}^{2}}{K^{2}}\right)^{3} \times \frac{1}{\left|V\left(u_{12}\right)\right|^{2}\left|V\left(u_{23}\right)\right|^{2}\left|V\left(u_{13}\right)\right|^{2}}
\end{aligned}
$$

where $K$ is the total number of detected photoevents, $N_{\text {tel }}$ the number of telescopes, $N_{\text {pix }}$ the number of pixels, and $\sigma_{r}$ the detector readout noise. And $\left|V\left(u_{12}\right)\right|,\left|V\left(u_{23}\right)\right|,\left|V\left(u_{13}\right)\right|$, are the visibility amplitudes of the observed object.

\section{A.2. Error on the parameters of a double system}

We want to estimate the errors on the parameters of a double system made of an extended uniform disk and a point source, from a least square fit of a set of $N_{\mathrm{c}}$ uncorrelated phase closure measurements obtained with an interferometer formed by $N_{\text {tel }}$ telescopes. For simplicity, we assume that the telescopes are aligned, with a non redundant configuration, and that the direction of observation varies little during the experiment and may be considered constant. Under these conditions, the object is characterized by 3 parameters: the stellar angular radius $R_{\star}$, the flux ratio $r$, and the projected separation $s$ along the direction of observation.

The $N_{\mathrm{c}}$ phase closures are sampled at frequencies $u_{l n}^{k}\{k=$ $\left.1, \ldots, N_{\mathrm{c}} ; \ln =12,23,13\right\}$, in frequency intervals $\Delta u_{l n}$. We define the $N_{\mathrm{c}}$ lines and 3 columns matrix $\mathcal{M}$, of element $m_{j k}$, by

$m_{j k}=\frac{1}{\sigma\left(\phi_{\mathrm{c}}\right)} \frac{\partial \phi_{\mathrm{c}}^{k}}{\partial p_{j}} \quad\left(\right.$ with $\left.\left\{p_{1}, p_{2}, p_{3}\right\}=\left\{R_{\star}, r, s\right\}\right)$.

The variances of the parameters are the diagonal elements of the matrix $\left[{ }^{t} \mathcal{M} \times \mathcal{M}\right]^{-1}$. Let us first examamine the matrix ${ }^{t} \mathcal{M} \times \mathcal{M}$. 
It is given by

$$
\left[\begin{array}{ccc}
\sum_{k} \frac{1}{\sigma_{k}^{2}}\left(\frac{\partial \phi_{\mathrm{c}}^{k}}{\partial R_{\star}}\right)^{2} & \sum_{k} \frac{1}{\sigma_{k}^{2}} \frac{\partial \phi_{\mathrm{c}}^{k}}{\partial R_{\star}} \frac{\partial \phi_{\mathrm{c}}^{k}}{\partial r} & \sum_{k} \frac{1}{\sigma_{k}^{2}} \frac{\partial \phi_{\mathrm{c}}^{k}}{\partial R_{\star}} \frac{\partial \phi_{\mathrm{c}}^{k}}{\partial s} \\
\sum_{k} \frac{1}{\sigma_{k}^{2}} \frac{\partial \phi_{\mathrm{c}}^{k}}{\partial R_{\star}} \frac{\partial \phi_{\mathrm{c}}^{k}}{\partial r} & \sum_{k} \frac{1}{\sigma_{k}^{2}}\left(\frac{\partial \phi_{\mathrm{c}}^{k}}{\partial r}\right)^{2} & \sum_{k} \frac{1}{\sigma_{k}^{2}} \frac{\partial \phi_{\mathrm{c}}^{k}}{\partial s} \frac{\partial \phi_{\mathrm{c}}^{k}}{\partial r} \\
\sum_{k} \frac{1}{\sigma_{k}^{2}} \frac{\partial \phi_{\mathrm{c}}^{k}}{\partial R_{\star}} \frac{\partial \phi_{\mathrm{c}}^{k}}{\partial s} & \sum_{k} \frac{1}{\sigma_{k}^{2}} \frac{\partial \phi_{\mathrm{c}}^{k}}{\partial s} \frac{\partial \phi_{\mathrm{c}}^{k}}{\partial r} & \sum_{k} \frac{1}{\sigma_{k}^{2}}\left(\frac{\partial \phi_{\mathrm{c}}^{k}}{\partial s}\right)^{2}
\end{array}\right]
$$

We neglect now the detector noise (photon noise regime), and we assume that we are working outside the null regions, so that the phase closure may be approximated by Eq. (7). Each element of the matrix above is the sum of nine terms. There are two types of terms

$$
\begin{aligned}
& A_{l n, p q}=\sum_{k} f\left(u_{l n}^{k}\right) \sin \left(2 \pi u_{l n}^{k} s\right) \cos \left(2 \pi u_{p q}^{k} s\right) \\
& B_{l n, p q}=\sum_{k} g\left(u_{l n}^{k}\right) \sin \left(2 \pi u_{l n}^{k} s\right) \sin \left(2 \pi u_{p q}^{k} s\right)
\end{aligned}
$$

where $f(u)$ and $g(u)$ are smoothly varying functions over the working frequency intervals $\Delta u_{l n}$.

At last, we assume that the projected separation $s$ is larger than a few stellar radii and that the working frequency intervals are larger than $s^{-1}$, but smaller than the mean frequencies:

$s>$ a few $R_{\star} \quad$ and $\quad \bar{u}_{l n} \gg \Delta u_{l n} \geq s^{-1}$.

Under these conditions, all the $A$ terms are equal to zero and, given that $u_{12} \neq u_{23} \neq u_{13}$ (non redundancy), the only non null $B$ terms are those for which $\{\ln \}=\{p q\}$. In addition,

$B_{l n, l n}=\sum_{k} g\left(u_{l n}^{k}\right) \sin ^{2}\left(2 \pi u_{l n}^{k} s\right) \approx \frac{N_{\mathrm{c}}}{2} \times \overline{g\left(u_{l n}\right)}$

where $\overline{g\left(u_{l n}\right)}$ is the average value of $g\left(u_{l n}\right)$ over the working frequency interval $\Delta u_{l n}$. The matrix ${ }^{t} \mathcal{M} \times \mathcal{M}$ may then be written into the form

${ }^{t} \mathcal{M} \times \mathcal{M} \approx \frac{K}{N_{\text {tel }}^{2}}\left[\begin{array}{ccc}r^{2} \overline{X\left(R_{\star}\right)} & r \overline{Y\left(R_{\star}\right)} & 0 \\ r \overline{Y\left(R_{\star}\right)} & 1 & 0 \\ 0 & 0 & \left(2 \pi \bar{u}_{\max } r\right)^{2} \overline{Z\left(R_{\star}\right)}\end{array}\right]$

where $K$ is the total number of photoevents detected during the whole observation and $\bar{u}_{\max }$ the average value of the highest frequency. $X\left(R_{\star}\right), Y\left(R_{\star}\right)$, and $Z\left(R_{\star}\right)$ are given by

$$
\begin{aligned}
& \overline{X\left(R_{\star}\right)}=\frac{\sum_{\ln } \frac{1}{V_{\star}^{4}\left(u_{l n}\right)}\left(\frac{\partial V_{\star}\left(u_{l n}\right)}{\partial R_{\star}}\right)^{2}}{\sum_{\ln } \frac{1}{V_{\star}^{2}\left(u_{l n}\right)}} \\
& \overline{Y\left(R_{\star}\right)}=\frac{\sum_{\ln \frac{1}{V_{\star}^{3}\left(u_{l n}\right)} \frac{\partial V_{\star}\left(u_{l n}\right)}{\partial R_{\star}}}^{\sum_{\ln } \frac{1}{V_{\star}^{2}\left(u_{l n}\right)}}}{\overline{Z\left(R_{\star}\right)}}=\frac{\frac{1}{\bar{u}_{\max }^{2}} \sum_{\ln } \frac{u_{l n}^{2}}{V_{\star}^{2}\left(u_{l n}\right)}}{\sum_{\ln } \frac{1}{V_{\star}^{2}\left(u_{l n}\right)}} .
\end{aligned}
$$

The errors on the parameters of the system may now be computed. Inverting the matrix ${ }^{t} \mathcal{M} \times \mathcal{M}$ provides

$$
\begin{aligned}
& \sigma^{2}\left(R_{\star}\right) \approx \frac{N_{\mathrm{tel}}^{2}}{r^{2} K} \times \frac{1}{\overline{X\left(R_{\star}\right)}-\overline{Y\left(R_{\star}\right)^{2}}} \\
& \sigma^{2}(r) \approx \frac{N_{\mathrm{tel}}^{2}}{K} \times \frac{\overline{X\left(R_{\star}\right)}}{\overline{X\left(R_{\star}\right)}-\overline{Y\left(R_{\star}\right)}}{ }^{2} \\
& \sigma^{2}\left(s / R_{\star}\right) \approx \frac{N_{\mathrm{tel}}^{2}}{r^{2} K} \times \frac{1}{\left(2 \pi \bar{u}_{\max } R_{\star}\right)^{2} \overline{Z\left(R_{\star}\right)}} .
\end{aligned}
$$

Let us focus on the flux ratio and on the stellar separation. Numerical calculations show that the quantities $\overline{X\left(R_{\star}\right)} /\left[\overline{X\left(R_{\star}\right)}-\right.$ \left.${\overline{Y\left(R_{\star}\right)}}^{2}\right]$ and $\overline{Z\left(R_{\star}\right)}$ are fairly constant and close to 1 in a wide range of experimental conditions, even for observations performed around the nulls. Then, in the photon noise regime, the errors on the flux ratio and the separation may be fairly approximated by

$$
\begin{aligned}
& \sigma(r) \approx \frac{N_{\mathrm{tel}}}{\sqrt{K}} \\
& \sigma\left(s / R_{\star}\right) \approx \frac{N_{\mathrm{tel}}}{r \sqrt{K}} \times \frac{1}{2 \pi \bar{u}_{\max } R_{\star}}
\end{aligned}
$$

Acknowledgements. This research has made use of the SearchCal service of the Jean-Marie Mariotti Centre ${ }^{1}$, of the CDS Astronomical Databases SIMBAD and VIZIER, and of the NASA Astrophysics Data System Abstract Service.

\section{References}

Benest, D., \& Duvent, J. L. 1995, A\&A, 299, 621

Bonneau, D., Clausse, J.-M., Delfosse, X., et al. 2006, A\&A, 456, 789 Bracewell, R. N. 1978, Nature, 274, 780

Charbonneau, D., Allen, L. E., Megeath, S. T., et al. 2005, ApJ, 626, 523 Chelli, A. 1989, A\&A, 225, 277

Del Rio, L., Ribiere, M., Delage, L., \& Reynaud, F. 2008, Opt. Commun., 281, 2722

Döllinger, M. P., Hatzes, A. P., Pasquini, L., et al. 2007, A\&A, 472, 649

Duvert, G., Chelli, A., Malbet, F., \& Kern, P. 2009, A\&A, in press

Fortney, J. J., Lodders, K., Marley, M. S., \& Freedman, R. S. 2008, ApJ, 678, 1419

Hatzes, A. P., \& Cochran, W. D. 1998, MNRAS, 293, 469

Hatzes, A. P., Guenther, E. W., Endl, M., et al. 2005, A\&A, 437, 743

Kern, P., Le Coarer, E., \& Benech, P. 2009, Opt. Express

Knutson, H. A., Charbonneau, D., Allen, L. E., Burrows, A., \& Megeath, S. T. 2008, ApJ, 673, 526

Le Bouquin, J.-B., Bauvir, B., Haguenauer, P., et al. 2008, A\&A, 481, 553

Le Coarer, E., Blaize, S., Benech, P., et al. 2007, Nature Photonics, 1, 473

Mayor, M., \& Queloz, D. 1995, Nature, 378, 355

Michelson, A. A., \& Pease, F. G. 1921, ApJ, 53, 249

Perrin, G., Woillez, J., Lai, O., et al. 2006, Science, 311, 194

Petrov, R. G., Malbet, F., Weigelt, G., et al. 2007, A\&A, 464, 1

Rothman, J., Perrais, G., Destefanis, G., et al. 2007, in SPIE Conf. Ser., 6542

Tatulli, E., \& Chelli, A. 2005, J. Opt. Soc. Am. A, 22, 1589

Traub, W. A., \& Jucks, K. W. 2002, A Possible Aeronomy of Extrasolar Terrestrial Planets (Atmospheres in the Solar System: Comparative Aeronomy), 369

Vannier, M., Petrov, R. G., Lopez, B., \& Millour, F. 2006, MNRAS, 367, 825

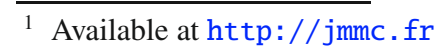

